\title{
Solar Sail Near-Optimal Circular Transfers with Plane Change
}

\author{
Giovanni Mengali and Alessandro A. Quarta \\ University of Pisa, I-56122 Pisa, Italy \\ DOI: $10.2514 / 1.38079$
}

\begin{abstract}
Near-minimum time interplanetary trajectories between circular orbits with different orbital planes are investigated. By assumption, the mission is carried out with a solar sail spacecraft, whose performance takes into account the optical characteristics of the sail film and the maximum temperature constraint. In an effort to obtain a general, albeit approximate, solution, the problem is tackled by dividing the mission in two or three phases, depending on the value of the inclination change between the initial and target orbit. Each phase is analyzed by solving a minimum time problem with an indirect approach, and the whole mission time is estimated as the sum of the contributions of the elementary phases. The obtained results are then collected through graphs and fitting functions that can be used effectively to get a good and quick estimate of the main mission parameters, as well as to quantify their effect on the mission flight time. A comparison of the proposed approach with the optimal results available for the Solar Polar Imager mission confirms the effectiveness of the developed methodology.
\end{abstract}

\section{Nomenclature}

$\begin{array}{ll}A & =\text { sail area } \\ a & =\text { semimajor axis } \\ a_{c} & =\text { sail characteristic acceleration } \\ a_{n}, a_{r}, a_{t} & =\text { normal, radial, tangential propulsive accelerations } \\ b_{1}, b_{2}, b_{3} & =\text { force coefficients } \\ e & =\text { orbital eccentricity } \\ f, g, h, k & =\text { modified equinoctial elements } \\ H & =\text { Hamiltonian } \\ i & =\text { orbital inclination } \\ \hat{i} & =\text { unit vector } \\ J & =\text { functional } \\ L & =\text { true longitude } \\ m & =\text { sailcraft mass } \\ n & =\text { number of revolutions } \\ p & =\text { semilatus rectum } \\ r & =\text { sun-spacecraft distance } \\ T & =\text { orbital period } \\ t & =\text { time } \\ w & =\text { auxiliary variable, see Eq. (7) } \\ \alpha & =\text { sail cone angle } \\ \beta_{\sigma} & =\text { dimensionless sail loading } \\ \Delta t & =\text { flight time } \\ \delta & =\text { sail clock angle } \\ \Theta & =\text { equilibrium temperature } \\ \lambda & =\text { adjoint variable } \\ \mu & =\text { gravitational parameter } \\ \nu & =\text { true anomaly } \\ \tilde{\sigma} & =\text { reference sail loading } \\ \Omega & =\text { right ascension of the ascending node } \\ \omega & =\text { argument of pericenter } \\ & \end{array}$

Received 15 April 2008; revision received 17 December 2008; accepted for publication 17 December 2008. Copyright ( 2008 by Giovanni Mengali and Alessandro A. Quarta. Published by the American Institute of Aeronautics and Astronautics, Inc., with permission. Copies of this paper may be made for personal or internal use, on condition that the copier pay the $\$ 10.00$ per-copy fee to the Copyright Clearance Center, Inc., 222 Rosewood Drive, Danvers, MA 01923; include the code 0731-5090/09 \$10.00 in correspondence with the CCC.

*Associate Professor, Department of Aerospace Engineering; g.mengali@ ing.unipi.it. Member AIAA.

'Research Assistant, Department of Aerospace Engineering; a.quarta@ ing.unipi.it. Senior Member AIAA.

$$
\begin{array}{lll}
\text { Subscripts } & \\
c & =\text { cranking } \\
f & =\text { final } \\
\lim & =\text { limit value } \\
\max & =\text { maximum } \\
\min & =\text { minimum } \\
0 & = & \text { initial } \\
\oplus & = & \text { Earth } \\
\odot & = & \text { sun } \\
\text { Superscripts } & \\
. & \\
\star & =\text { time derivative } \\
& = & \text { critical value }
\end{array}
$$

\section{Introduction}

$\mathbf{T}$ HE great interest of the scientific community in solar sails is closely related to their capability of generating thrust without the need of any propellant, a fundamental feature which is especially useful for long duration interplanetary missions $[\underline{1}, 2]$. In fact, the capability of using the solar radiation pressure for creating propulsive thrust allows solar sails to perform trajectories with large changes in orbital energy without the need of resorting to intermediate flyby maneuvers with celestial bodies.

A promising application of solar sails is connected to the achievement of heliocentric circular orbits with small radius and high inclination. Many of those missions are of great scientific relevance, such as missions to near-Earth objects with highly inclined orbits [3]. Another interesting example is represented by the Solar Polar Imager (SPI) mission, one of several sun-Earth connection solar sail roadmap missions currently envisioned by NASA [4,5]. The SPI mission schedules the insertion of a spacecraft into a heliocentric circular orbit having radius $0.48 \mathrm{AU}$ (astronomical unit) and inclination $75 \mathrm{deg}$. This mission is intended to obtain measurements of the solar magnetic field, the coronal mass ejections, and the solar irradiance in the sun's polar regions. Such information represents a crucial step to understanding the solar magnetic dynamo.

Technological constraints, essentially due to both the weight and the characteristic dimensions of a solar sail, limit the producible thrust to rather small values. Currently, the characteristic acceleration (that is, the maximum propelling acceleration at $1 \mathrm{AU}$ from the sun) does not exceed some fraction of a millimeter per second squared. These values entail long transfer times. For example, in a recent study Dachwald et al. [6] have shown that a square solar 
sail with a side length of $160 \mathrm{~m}$ and a characteristic acceleration of $0.35 \mathrm{~mm} / \mathrm{s}^{2}$ requires a flight time of 4.7 years to transfer the sailcraft on the SPI working orbit.

The study and the optimization phase of a solar sail trajectory are usually a rather complex procedure. The classical approach consists of using either a direct optimization method [7-9] or an indirect method [10-12], to look for optimal (minimum time) trajectories. However, apart from the particular technique used in the solution process, the calculation volume necessary to find an optimal trajectory is usually considerable, due to the great number of revolutions around the sun required by the sail to reach the final orbit. This problem is further worsened when a high variation of orbital inclination is sought [13] or when the sail characteristic acceleration is very small [14]. In fact, when an indirect method is employed in the optimization process, the long simulation time makes the solution a difficult task especially because the boundary values are extremely sensitive to the initial guess value of the adjoint variables. On the other hand, a direct method has other disadvantages, as the whole trajectory requires a high number of parameters for the discretization process. From this point of view the SPI mission offers a particularly representative example of these difficulties, due to the high inclination of the final orbit. Note that a reasonable estimate of the main mission parameters as, for example, the total flight time or the minimum distance from the sun during the transfer, is of primary importance for initializing the optimization process based on a direct approach $[\underline{1}, 15]$. Usually, a preliminary estimate of these parameters is obtained by dividing the mission in a certain number of phases and by using, in each phase, a locally optimal control law [6,13]. The aim is to maximize, at any time, one of the classical orbital parameters, or their combination, depending on the mission requirements. The strength of such control laws lies in their capability of approximating the optimal trajectory through simple numerical simulations. Moreover, these control laws are particularly useful for an analysis of planetocentric orbits [16], especially as long as escape missions are concerned $[17,18]$, due to their capability of including the main perturbation sources in the simulation process. However, an approach based on locally optimal control laws is not well suited for the particular problem we are interested in. A first intrinsic limit is related to the difficulty of satisfying the problem boundary conditions within reasonable tolerances. In our mission scenario these conditions are constituted by the final radius and the orbital inclination. A possible solution would be that of using the so-called blended, locally optimal control laws $[16,19]$. However, with such an approach, other parameters (the control weights) must be introduced to properly combine different locally optimal control laws. In principle, a suitable choice of these parameters allows the boundary conditions to be met. Nevertheless, this result is obtained at the expense of a further complication of the whole approach, as the choice of the weights requires some trial and error procedure. Moreover, the use of blended, locally optimal control laws hardly enables a generalization of the numerical results in terms of simple formulas relating mission performance (that is, flight time and minimum sun distance) with problem geometry (initial and final orbital radii and inclinations).

The aim of this paper is to provide a flexible tool, useful for a preliminary mission analysis, capable of providing a reasonable estimate of the main mission parameters with minor computational effort. The obtained results may then be used effectively as a first guess for more refined methods and as a guide for finding true globally optimal trajectories. The proposed approach seeks to combine the strength of a locally optimal method (in terms of simplicity and effectiveness) with that of a globally optimal approach (fulfilment of boundary constraints and solution reliability) to produce an approximate solution in the form of graphs and interpolating functions. To this end, the whole mission is first divided into two or three phases depending on the target orbit characteristics. Each phase is then studied in a global optimal framework using an indirect approach, and the final trajectory is simply obtained as an orderly sequence of the different phases. The rationale is that each phase is, by choice, univocally identified through a small number of parameters and, therefore, a parametric characterization of the results is possible.

\section{Problem Statement}

Consider the problem of a minimum time heliocentric transfer of a sailcraft from an initial circular orbit of radius $r_{0}$ and inclination $i_{0}$ to a second circular orbit with radius $r_{f}$ and inclination $i_{f}$ (without loss of generality assume that $r_{f}<r_{0}$ ). This problem is now approximated through a simplified model, with the aim of obtaining a compact and near-optimal solution. The main idea consists of taking advantage from the solar radiation pressure increase by performing the cranking maneuver as close as possible to the sun, depending on the maximum allowable temperature of the sail film. This strategy is met by dividing the mission into three phases: approach, cranking, and raising phases. In the first one (approach phase), the sailcraft is transferred, in a time interval $\Delta t_{1}$, from the starting heliocentric orbit to a circular intermediate orbit (referred to as the cranking orbit) with radius $r_{c} \leq r_{f}$ and inclination $i_{c} \equiv i_{0}$. Without loss of generality, due to the problem spherical symmetry, an initial inclination $i_{0}=0$ is assumed. In this phase the sailcraft follows a trajectory coplanar to the starting orbit whose complexity (in terms of number of revolutions around the sun and flight time) depends on the sail characteristic acceleration and on the ratio $r_{c} / r_{0}$.

The second phase (cranking phase) is used to vary the orbital plane position only, by maintaining a nearly constant distance from the sun $\left(r \cong r_{c}\right.$ ) while driving the orbital inclination to the desired final value $i_{f}$. The time length of the cranking phase, $\Delta t_{2}$, is rather long (often comparable to that of phase 1) because the sail propelling acceleration is effective in a restricted orbital region, that is, near the orbital nodes [16]. The value of $\Delta t_{2}$ depends on the sailcraft characteristic acceleration, the required orbital inclination $i_{f}$, and the local solar radiation pressure (i.e., on $r_{c}$ ).

In the third and final phase (raising phase) the orbital radius is increased, while maintaining the orbital inclination unchanged, up to the achievement of the desired value $r_{f}$, in a time interval $\Delta t_{3}$. The value of $\Delta t_{3}$ is a function of $a_{c}$ and $r_{c} / r_{f}$. Because the conceptual scheme of the third phase is identical to that of phase 1 , the solar sail performance of both phases 1 and 3 will be analyzed together. The total mission time is simply the sum of the times corresponding to the three single phases, that is, $\Delta t=\Delta t_{1}+\Delta t_{2}+\Delta t_{3}$.

The minimum admissible value for the cranking radius $r_{c}$ is typically constrained by structural requirements and, in particular, by the temperature limit of the sail film. Note that the cranking phase is onerous in terms of flight time, especially when inclination variations of some tens of degrees are required. Therefore, it is convenient to perform the cranking maneuver when the solar distance is as small as possible, in order to maximize the sail thrust that varies as the inverse square solar distance. On the other hand, as $r_{c}$ is reduced, the sail film equilibrium temperature tends to increase, and it may eventually exceed the maximum allowable value of either the payload or the solar sail bearing structure [20,21]. However, the solar sail equilibrium temperature depends not only on the optical characteristics of the reflective film (that are a function of the solar distance), but also on the sail pitch angle $[1,22]$. Therefore, once the maximum admissible temperature is fixed, there exists a maximum allowable pitch angle for a given distance from the sun. The existence of such an upper value provides an additional constraint that must be properly taken into account during the performance evaluation process.

The above problem of heliocentric transfer is now discussed assuming a flat solar sail whose performance takes into account the optical characteristics of the reflecting sail film through the so-called optical force model [1].

\section{Mathematical Model}

The equations of motion for a flat solar sail, of mass $m$ and area $A$, in a Cartesian heliocentric inertial frame can be written in terms of modified equinoctial elements [23-26] as 


$$
\dot{p}=(2 p / w) \sqrt{p / \mu_{\odot}} a_{t}
$$

$$
\begin{aligned}
\dot{f} & =\sqrt{p / \mu_{\odot}}\left\{a_{r} \sin L+[(w+1) \cos L+f]\left(a_{t} / w\right)\right. \\
& \left.-(h \sin L-k \cos L)\left(g a_{n} / w\right)\right\}
\end{aligned}
$$

$$
\begin{aligned}
\dot{g} & =\sqrt{p / \mu_{\odot}}\left\{-a_{r} \cos L+[(w+1) \sin L+g]\left(a_{t} / w\right)\right. \\
& \left.+(h \sin L-k \cos L)\left(f a_{n} / w\right)\right\}
\end{aligned}
$$

$$
\dot{h}=\sqrt{p / \mu_{\odot}}\left[a_{n}\left(1+h^{2}+k^{2}\right) /(2 w)\right] \cos L
$$

$$
\dot{k}=\sqrt{p / \mu_{\odot}}\left[a_{n}\left(1+h^{2}+k^{2}\right) /(2 w)\right] \sin L
$$

$$
\dot{L}=\sqrt{\mu_{\odot} p}(w / p)^{2}+\sqrt{p / \mu_{\odot}}(h \sin L-k \cos L) a_{n} / w
$$

where

$$
w \triangleq 1+f \cos L+g \sin L
$$

while $a_{r}, a_{t}$, and $a_{n}$ are the components of the solar sail propelling acceleration in a rotating radial-tangential-normal $\mathcal{T}_{R T N}\left(\hat{\boldsymbol{i}}_{R}, \hat{\boldsymbol{i}}_{T}, \hat{\boldsymbol{i}}_{N}\right)$ coordinate frame [25], given by

$$
\begin{gathered}
a_{r}=\frac{\beta_{\sigma} \mu_{\odot}}{2(p / w)^{2}} \cos \alpha\left[b_{1}+\left(b_{2} \cos \alpha+b_{3}\right) \cos \alpha\right] \\
a_{t}=\frac{\beta_{\sigma} \mu_{\odot}}{4(p / w)^{2}} \sin (2 \alpha) \cos \delta\left(b_{2} \cos \alpha+b_{3}\right) \\
a_{n}=\frac{\beta_{\sigma} \mu_{\odot}}{4(p / w)^{2}} \sin (2 \alpha) \sin \delta\left(b_{2} \cos \alpha+b_{3}\right)
\end{gathered}
$$

In the preceding equations the dimensionless sail loading [27] is defined as $\beta_{\sigma} \triangleq \tilde{\sigma} /(m / A)$, with $\tilde{\sigma} \triangleq 1.539 \mathrm{~g} / \mathrm{m}^{2}$. The cone angle $\alpha \in[0, \pi / 2]$ is the angle between the sun line vector and the sail normal vector (the latter being directed away from the sun). The sail clock angle $\delta \in[0,2 \pi]$, which describes the sail rotational orientation about the sun line vector, is defined as the angle between the sail normal vector projection onto the $\left(\hat{\boldsymbol{i}}_{T}, \hat{\boldsymbol{i}}_{N}\right)$ plane and the $\hat{\boldsymbol{i}}_{T}$ axis. Because $\alpha$ and $\delta$ unambiguously characterize the sail orientation with respect to the incoming solar radiation, they constitute the two sailcraft control variables. Assuming a solar sail with a highly reflective aluminum-coated front side and a highly emissive chromium-coated backside, the force coefficients [12] are $b_{1}=0.1728, b_{2}=1.6544$, and $b_{3}=-0.0109$. The employment of modified equinoctial elements in the equations of motion is useful for obtaining a significant reduction in the computational time necessary to find the solar sail trajectory [25].

A relationship exists between the dimensionless sail loading $\beta_{\sigma}$ and the characteristic acceleration $a_{c}$, the latter being the parameter commonly used to quantify the performance of a solar sail. If one thinks of $a_{c}$ as the maximum sail propulsive acceleration (corresponding to $\alpha=0$ ) when the sun-sailcraft distance is $r=r_{\oplus} \triangleq 1 \mathrm{AU}$, then, recalling that $p / w=r[\underline{23}]$, from Eqs. $(\underline{8}-\underline{10})$ one obtains

$$
a_{c}=\beta_{\sigma} \frac{\mu_{\odot}}{r_{\oplus}^{2}} \frac{\left(b_{1}+b_{2}+b_{3}\right)}{2}
$$

where $\mu_{\odot} / r_{\oplus}^{2} \cong 5.930 \mathrm{~mm} / \mathrm{s}^{2}$ is the sun's gravitational acceleration at $1 \mathrm{AU}$ distance.

The transformations from modified equinoctial elements to classical orbital elements are obtained from the following relationships:

$$
a=\frac{p}{1-f^{2}-g^{2}}
$$

$$
e=\sqrt{f^{2}+g^{2}}
$$

$$
i=2 \arctan \sqrt{h^{2}+k^{2}}
$$

$$
\sin \omega=g h-f k, \quad \cos \omega=f h+g k
$$

$$
\sin \Omega=k, \quad \cos \Omega=h
$$

$$
v=L-\Omega-\omega
$$

The minimum time necessary to complete the transfer between circular orbits with different orbital planes can be approximated by solving the near-optimal transfer problem described in the previous section, that is, by calculating the minimum times $\Delta t_{1}, \Delta t_{2}$, and $\Delta t_{3}$. The latter problem, in its turn, can be solved using an indirect approach [28]. To this end consider the Hamiltonian $H$ of the system, given by [see Eqs. (1-6)]

$$
H \triangleq \lambda_{p} \dot{p}+\lambda_{f} \dot{f}+\lambda_{g} \dot{g}+\lambda_{h} \dot{h}+\lambda_{k} \dot{k}+\lambda_{L} \dot{L}
$$

where $\lambda_{p}, \lambda_{f}, \lambda_{g}, \lambda_{h}, \lambda_{k}$, and $\lambda_{L}$ are the adjoint variables. The optimal value of the control angles $\alpha$ and $\delta$ is obtained by invoking Pontryagin's maximum principle, that is, by maximizing $H$ at any time. As long as the clock angle $\delta$ is concerned, by imposing the necessary condition $\partial H / \partial \delta=0$ one has

$$
\begin{gathered}
\sin \delta=\left[\lambda_{k}\left(1+h^{2}+k^{2}\right)+2 h\left(\lambda_{L}+\lambda_{g} f-\lambda_{f} g\right)\right] \sin L \\
+\left[\lambda_{h}\left(1+h^{2}+k^{2}\right)-2 k\left(\lambda_{L}+\lambda_{g} f-\lambda_{f} g\right)\right] \cos L \\
\cos \delta=4 \lambda_{p} p+3 \lambda_{f} f+3 \lambda_{g} g+\left(\lambda_{f} f-\lambda_{g} g\right) \cos 2 L \\
+\left(\lambda_{g} f+\lambda_{f} g\right) \sin 2 L+4 \lambda_{g} \sin L+4 \lambda_{f} \cos L
\end{gathered}
$$

On the other hand, the other necessary condition $\partial H / \partial \alpha=0$ does not provide a closed-form solution for the cone angle $\alpha$. Therefore, the maximization of $H$ with respect to $\alpha$ is performed by means of a numerical algorithm based on a golden section search and parabolic interpolation [29].

The time derivative of the $j$ th adjoint variable is obtained from the Euler-Lagrange equations:

$$
\dot{\lambda}_{j}=-\frac{\partial H}{\partial j} \quad \text { with } \quad j \triangleq\left(\lambda_{p}, \lambda_{f}, \lambda_{g}, \lambda_{h}, \lambda_{k}, \lambda_{L}\right)
$$

The explicit expression of the Euler-Lagrange equations is rather involved and is not reported here for the sake of conciseness. The boundary-value problem associated with the variational problem is constituted by the six equations of motion (1-6) and the six EulerLagrange equations (21). The corresponding 12 boundary conditions vary according to the phase number, as discussed in the next section. 


\section{Near-Optimal Circular Transfers}

Having outlined the approach used in the mission analysis, we are now in a position to analyze the single phases and to collect the corresponding results.

\section{Phase 1}

Let $t_{0}=0$ be the initial mission time. To minimize the time interval $\Delta t_{1}$ necessary to complete this first phase, the functional

$$
J_{1} \triangleq-\Delta t_{1}=t_{0}-t_{1} \equiv-t_{1}
$$

must be maximized. A suitable system of canonical units is now introduced for convenience. The distance unit $\mathrm{DU}_{1}$ coincides with the initial orbital radius $r_{0}$, while the time unit $\mathrm{TU}_{1}$ is chosen such that the solar gravitational parameter $\mu_{\odot}$ is unitary. With such a choice, recalling that the transfer orbit in this phase is coplanar to the initial orbit (whose inclination is, by assumption, $i_{0}=0$ ), the corresponding boundary conditions are [see Eqs. (12-17)]

$$
\begin{gathered}
p\left(t_{0}\right)=1 \mathrm{DU}_{1}, \quad f\left(t_{0}\right)=g\left(t_{0}\right)=h\left(t_{0}\right)=k\left(t_{0}\right)=L\left(t_{0}\right)=0 \\
p\left(t_{1}\right)=\left(r_{c} / r_{0}\right) \mathrm{DU}_{1} \\
f\left(t_{1}\right)=g\left(t_{1}\right)=h\left(t_{1}\right)=k\left(t_{1}\right)=\lambda_{L}\left(t_{1}\right)=0
\end{gathered}
$$

In Eq. (23) the condition $L\left(t_{0}\right)=0$ is chosen in view of problem symmetry, whereas the condition $\lambda_{L}\left(t_{1}\right)=0$ comes from the fact that the sailcraft position on the circular final orbit is left free. The optimal flight time is obtained by enforcing the transversality condition $H\left(t_{1}\right)=1$.

The optimal control problem has been solved for different values of the pair $\left(a_{c}, r_{c} / r_{0}\right)$ and the results are summarized in Fig. 1 . The transfer time $\Delta t_{1}$ is made dimensionless by dividing it by the initial orbital period $T_{0}=2 \pi \mathrm{TU}_{1}$. To quantify the sensitivity of the mission time on the characteristic acceleration, assume a baseline value $a_{c} \triangleq 0.35 \mathrm{~mm} / \mathrm{s}^{2}$, corresponding to the value selected for the SPI mission [6]. From Fig. 1 one obtains that, as long as $r_{c} / r_{0}<0.7$, the flight time doubles as $a_{c}^{-}$is halved and, vice versa, $\Delta t_{1}$ halves as $a_{c}$ is doubled. This result implies a great sensitivity of $\Delta t_{1}$ with respect to both $a_{c}$ and $r_{c} / r_{0}$.

The presence of ripples in the curves of flight time in Fig. 1 is associated with a condition where the sailcraft attains an integer number of revolutions around the sun before reaching the target circular orbit (this is confirmed by the dashed curves in Fig. 1). This

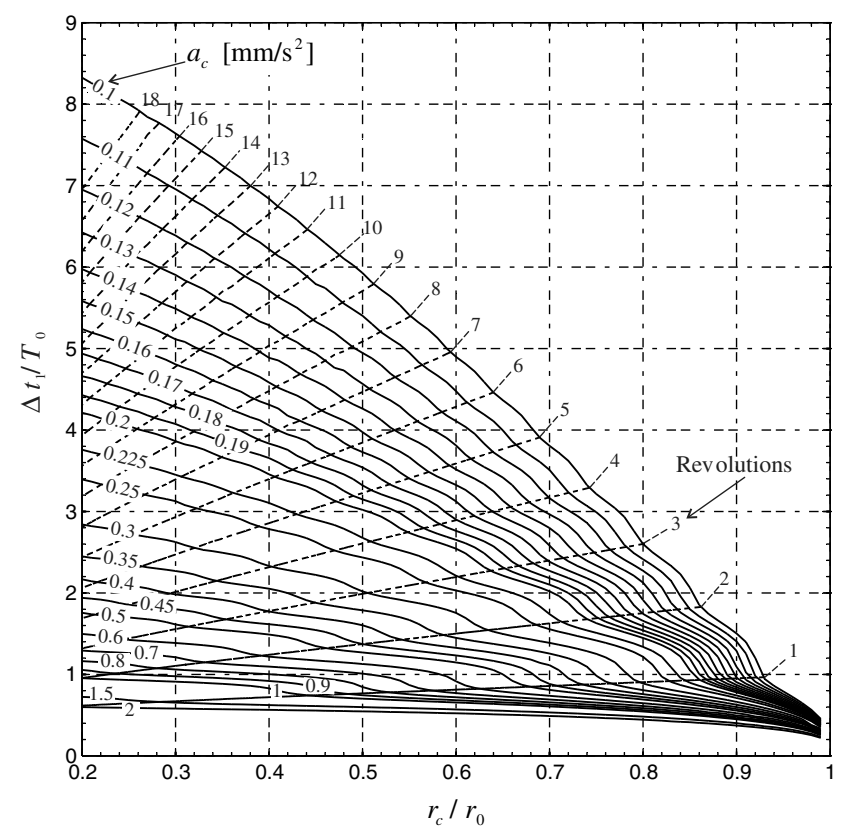

Fig. 1 Minimum flight time for phase 1 as a function of $a_{c}$ and $\left(r_{c} / r_{0}\right)$. behavior is similar to that found by Alfano and Thorne in [30] for the propellant consumption in a low-thrust circle-to-circle rendezvous.

\section{Phase 2}

Recall that the aim of this cranking phase is to rotate the orbital plane as quickly as possible, by maintaining a nearly fixed distance from the sun. For a given orbital radius $r_{c}$, the minimum time $\Delta t_{2} \triangleq t_{2}-t_{1}$ necessary for this phase is a function of the desired orbital inclination $i_{f}$. A set of canonical units is introduced, characterized by $\mathrm{DU}_{2} \triangleq r_{c}$ and $\mathrm{TU}_{2} \triangleq \sqrt{r_{c}^{3} / \mu_{\odot}}$. This allows one to make the simulation results of this phase independent of the cranking radius. To simplify the computation of $\Delta t_{2}$, the original minimum time problem is slightly modified and the maximum orbital inclination $\Delta i_{\max }$ (in a given reference time) is sought first. Because of the high number of revolutions necessary for the phase completion, the reference time is set equal to the period of the cranking orbit $T_{c}=2 \pi \mathrm{TU}_{2}$, and the corresponding value of $\Delta i_{\max }$ is found as a function of the characteristic acceleration only. The calculation proceeds as follows. Let $n$ be the complete integer number of revolutions during phase 2 , then the near-minimum cranking time is

$$
\Delta t_{2}=n T_{c}+\widetilde{\Delta t} \quad \text { with } \quad n \triangleq\left\lfloor i_{f} / \Delta i_{\max }\right\rfloor
$$

where $\lfloor\cdot\rfloor$ is the floor function and $\widetilde{\Delta t}<T_{c}$ is the remainder of the division $\Delta t_{2} /\left(n T_{c}\right)$. This approach allows one to substantially simplify the numerical calculations, because the propagation of the optimal solution is confined to a small time interval (equal to $T_{c}$ ). Moreover, this strategy has an interesting practical implication. In fact, once the optimal steering law for a complete orbital period is found, this control law can be repeated exactly alike $n$ times. The corresponding final inclination will be close to the desired value, and the remaining difference can be eliminated through the additional subphase whose length is $\widetilde{\Delta t}$, as indicated by Eq. (24).

The value of $\Delta i_{\max }$ can be found by solving an optimal control problem, aimed at maximizing the orbital inclination in the time interval $\left[t_{1}, t_{1}+T_{c}\right]$. Recalling the relationship (14) between the orbital inclination $i$ and the modified equinoctial elements $h$ and $k$, the 12 boundary conditions for the differential problem become

$$
\begin{gathered}
p\left(t_{1}\right)=1 \mathrm{DU}_{2}, \quad f\left(t_{1}\right)=g\left(t_{1}\right)=h\left(t_{1}\right)=k\left(t_{1}\right)=L\left(t_{1}\right)=0 \\
p\left(t_{1}+T_{c}\right)=1 \mathrm{DU}_{2} \\
f\left(t_{1}+T_{c}\right)=g\left(t_{1}+T_{c}\right)=\lambda_{L}\left(t_{1}+T_{c}\right)=0 \\
\lambda_{h}\left(t_{1}+T_{c}\right)=2 h\left(t_{1}+T_{c}\right), \quad \lambda_{k}\left(t_{1}+T_{c}\right)=2 k\left(t_{1}+T_{c}\right)
\end{gathered}
$$

The optimal problem has been solved for different values of the characteristic acceleration and the corresponding results are illustrated in Fig. 2. The curve shown in Fig. 2 can be approximated, with errors less than $3 \times 10^{-2} \mathrm{deg}$, through a second-order polynomial function in the form:

$$
\Delta i_{\max } \cong 13.73 a_{c}+0.07418 a_{c}^{2}
$$

where $\Delta i_{\max }$ is expressed in degrees and $a_{c}$ in $\mathrm{mm} / \mathrm{s}^{2}$. This result allows one to quickly and accurately obtain an estimate of the number of revolutions necessary for the cranking maneuver. For example, using the SPI mission as a reference case $\left(a_{c}=0.35 \mathrm{~mm} / \mathrm{s}^{2}\right)$, Eq. (26) implies that $\Delta i_{\max } \cong 4.82 \mathrm{deg}$. Recalling that $i_{f}=75 \mathrm{deg}$, the complete number of revolutions is $n=\lfloor 75 / 4.82\rfloor=15$. This result coincides with the solution found in [6] using a locally optimal steering law.

With the exception of the negligible term $\widetilde{\Delta t}$, Eq. (24) states that the total cranking time can be reduced by shortening either $n$ or $T_{c}$ (or both). The value of $n$ is proportional to the reciprocal of $\Delta i_{\max }$, the latter being independent of $r_{c}$ as a consequence of the boundary conditions (25). The other contribution affecting the cranking time is $T_{c}$ which, instead, depends on $r_{c}$ through the time unit $\mathrm{TU}_{2}$. Although small values of $r_{c}$ tend to reduce the value of $T_{c}$, however 


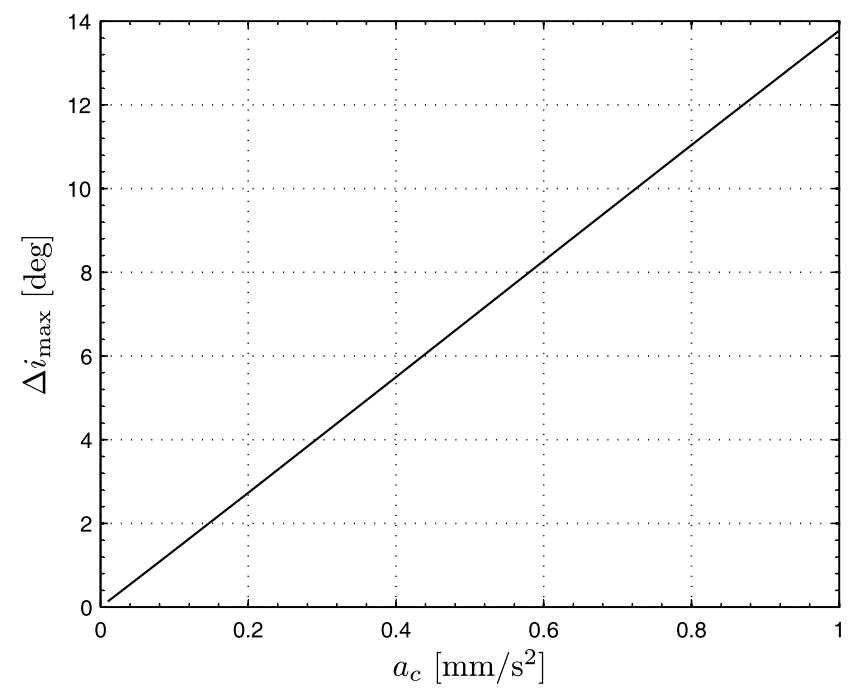

Fig. 2 Optimal cranking performance $\Delta i_{\max }$ as a function of $a_{c}$.

$r_{c}$ cannot be decreased under a minimum value, to avoid the fact that the system temperature may exceed the maximum tolerable limit. In fact, the sail equilibrium temperature is a function of both the sun distance and the sail cone angle as [6]

$$
\Theta=\widetilde{\Theta} \sqrt[4]{\cos \alpha} \sqrt{\frac{r_{\oplus}}{r}}
$$

where $\tilde{\Theta}$ is a reference temperature depending on the optical properties of the reflecting material $(\tilde{\Theta}=263.56 \mathrm{~K}$ for the model used in this paper) and $r$ is the local sun-sailcraft distance, see Fig. 3 . Assuming that the sail plane is orthogonal to the sun's rays $(\alpha=0)$, for a given sail film maximum tolerable limit $\Theta_{\text {lim }}$ Eq. (27) allows one to calculate the minimum allowable value of $r$. For example, if $\Theta_{\lim }=513.15 \mathrm{~K}\left(240^{\circ} \mathrm{C}\right)$, see [6], the minimum solar distance corresponding to $\alpha=0$ is 0.264 AU. Actually, the sail film may tolerate lower distances than the above value, provided the sail cone angle is greater than $\alpha=0$. Therefore, in the mission analysis it is preferable to directly take into account the maximum achieved temperature $\Theta_{\max }$ rather than $\Theta_{\text {lim }}$, where $\Theta_{\max } \triangleq \Theta\left(\alpha=\alpha_{\text {min }}\right) \leq$ $\Theta_{\lim }$ and $\alpha_{\min }$ is the minimum cone angle achieved during the cranking maneuver. The latter can be calculated numerically as a function of $a_{c}$, and the corresponding simulation results are summarized in Fig. 4. For characteristic accelerations $a_{c} \in[0.01,1] \mathrm{mm} / \mathrm{s}^{2}$, the minimum cone angle ranges in the

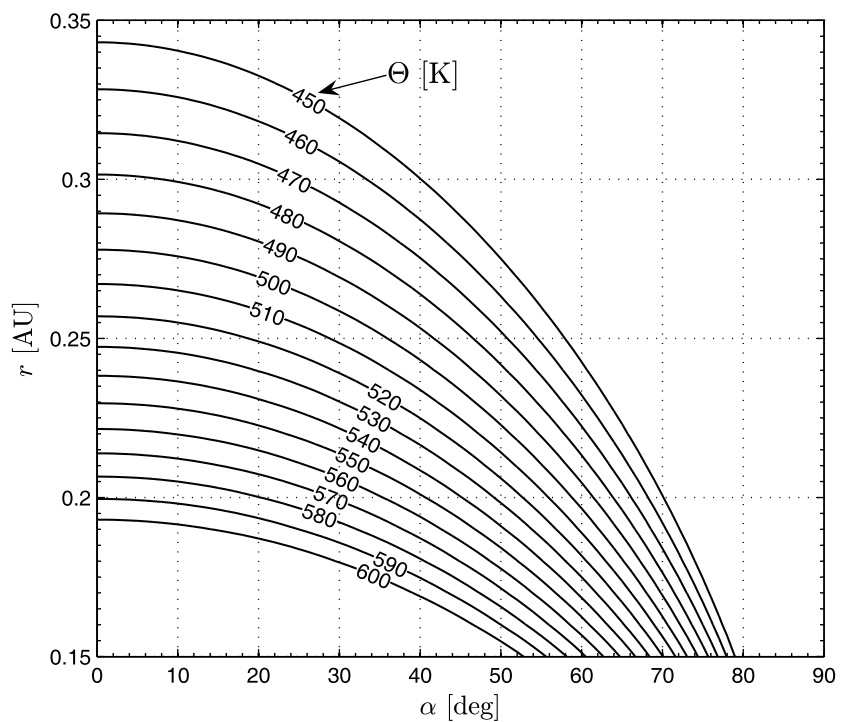

Fig. 3 Sailcraft equilibrium temperature $\Theta$ as a function of $r$ and $\alpha$. interval $[31,36] \mathrm{deg}$, in accordance with the values extrapolated from [6]. Therefore, $\alpha_{\min }$ is nearly independent of the characteristic acceleration, especially as long as values compatible with the current technology are examined $\left(a_{c}<0.5 \mathrm{~mm} / \mathrm{s}^{2}\right)$. When the data taken from Fig. $\underline{4}$ are inserted into Eq. (27), a relationship between $\Theta_{\max }$ and the distance $r_{c}$ is obtained. This relationship is drawn in Fig. 5 for two very different values of characteristic acceleration, that is, $\Theta_{\max }=\Theta_{\max }\left(a_{c}=0.01 \mathrm{~mm} / \mathrm{s}^{2}, r_{c}\right)$ and $\Theta_{\max }=\Theta_{\max }\left(a_{c}=\right.$ $\left.1 \mathrm{~mm} / \mathrm{s}^{2}, r_{c}\right)$. As expected from the previous analysis $\left(\alpha_{\min }\right.$ is nearly independent of $a_{c}$ ), the two curves are very close. Recalling Eq. (27), the curve of Fig. 5 can be approximated, with an error less than $1 . \overline{2 \%}$, through the following relationship:

$$
r_{c} \cong 0.9113 r_{\oplus}\left(\frac{\tilde{\Theta}}{\Theta_{\max }}\right)^{2}
$$

With reference, again, to the SPI mission $\left(\Theta_{\max }=513.15 \mathrm{~K}\right)$, from Eq. (28) the minimum cranking orbit distance is $r_{c} \cong 0.24 \mathrm{AU}$. This result differs from the global optimal value of $0.22 \mathrm{AU}$, found by Dachwald et al. [6], of less than $10 \%$.

According to Eq. (24) the total cranking time $\Delta t_{2}$ needs the calculation of $\widetilde{\Delta t} \in\left(0, T_{c}\right)$, that is, the time for completing the fraction of revolution required to obtain the desired inclination. For a solar distance $r_{c}$ and a given value of $a_{c}$, the maximum achievable variation of orbital inclination in the time interval $\widetilde{\Delta t}$ is

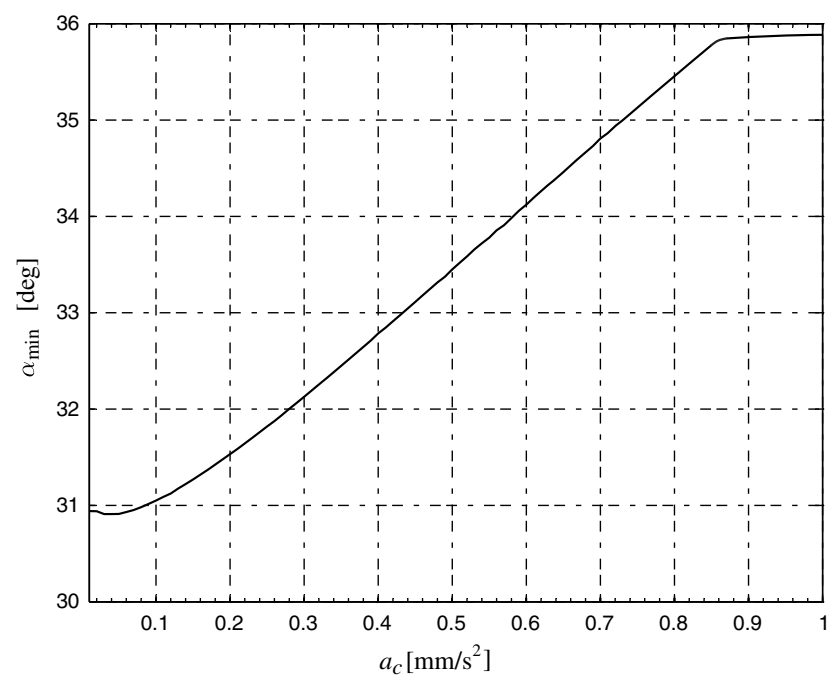

Fig. 4 Minimum cone angle $\alpha_{\min }$ during the cranking phase.

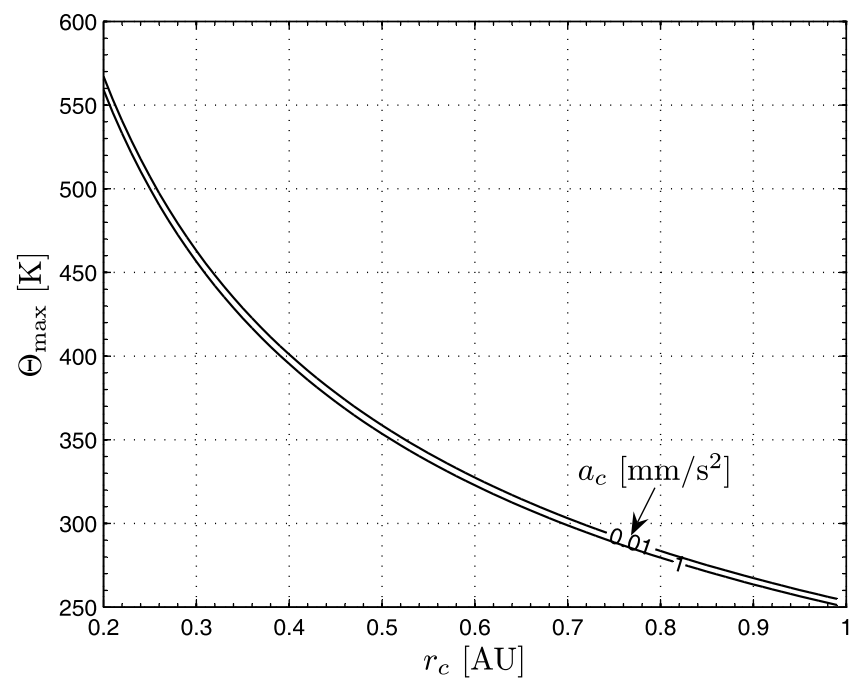

Fig. 5 Maximum sail temperature as a function of $\boldsymbol{r}_{c}$. 
$\widetilde{\Delta i}<\Delta i_{\max }\left(a_{c}\right)$. The relationship $\widetilde{\Delta i}=\widetilde{\Delta i}\left(a_{c}, \widetilde{\Delta t}\right)$ can be obtained numerically by solving an optimal problem formally identical to that previously analyzed in this section, the only difference being that the required flight time is now less than the orbital period $T_{c}$.

The simulation results are shown in Fig. 6, where the time interval $\widetilde{\Delta t}$ and the variation of orbital inclination $\widetilde{\Delta i}$ are normalized by $T_{c}$ and $\Delta i_{\max }\left(a_{c}\right)$, respectively. From Fig. 6 one concludes that, irrespective of the value of $a_{c}, \Delta i_{\max }$ is very small as long as the flight time is less than about $15 \%$ of the orbital period $T_{c}$. Moreover, all of the curves drawn for a given value of $a_{c} \in[0.1,1] \mathrm{mm} / \mathrm{s}^{2}$ are close to each other, with differences less than $10 \%$. Assuming $a_{c}=$ $0.35 \mathrm{~mm} / \mathrm{s}^{2}$ as a reference value, the different curves shown in Fig. 6 can be reasonably approximated through a single function independent of $a_{c}$ as

$$
\frac{\widetilde{\Delta t}}{T_{c}}=\frac{1}{2}\left[\left(\widetilde{\Delta i} / \Delta i_{\max }\right)^{2.152}+\left(\widetilde{\Delta i} / \Delta i_{\max }\right)^{0.219}\right]
$$

In summary, once the mission parameters $\left(a_{c}, \Theta_{\max }, i_{f}\right)$ are given, the flight time $\Delta t_{2}$ can be calculated as follows. From Fig. $\underline{5}$ [equivalently from Eq. (28)] one calculates the cranking radius $\bar{r}_{c}$ (that is, the time unit $\mathrm{TU}_{2}$ ) that meets the constraint on the maximum admissible temperature $\Theta_{\max }$. From Fig. 2, or from the approximate relationship (26), one obtains the maximum variation of orbital inclination $\Delta \overline{i_{\max }}$ in an orbital period $T_{c}$ corresponding to the given $a_{c}$. The complete number of revolutions is then obtained as $n=\left\lfloor i_{f} / \Delta i_{\max }\right\rfloor$, while $\widetilde{\Delta i}=i_{f}-n \Delta i_{\max }$. The time interval $\widetilde{\Delta t}$ is given by Eq. (29) or by Fig. $\underline{6}$. Finally, Eq. (24) provides the value of $\Delta t_{2}$.

\section{Phase 3}

In this phase the sailcraft performs a two-dimensional transfer between a circular orbit with radius $r_{c}$ and the final circular orbit with radius $r_{f}>r_{c}$. The evaluation of the minimum time interval $\Delta t_{3}$ necessary to complete this phase follows the same approach as that described for phase 1 . Because of symmetry considerations and in accordance with the results found in the literature $[31,32]$ it can be verified that, $a_{c}$ being the same, $\Delta t_{3}=\Delta t_{1}$ when $\left(r_{c} / r_{0}\right)=\left(r_{c} / r_{f}\right)$. This result is confirmed also by all of our numerical simulations. Therefore, the minimum time $\Delta t_{3}$ can be obtained with the aid of Fig. 1 by simply substituting the axis labels $\Delta t_{1}$ with $\Delta t_{3}, r_{0}$ with $r_{f}$, and $\bar{T}_{0}$ with $T_{f}$, where $T_{f}$ is the period of the circular final orbit.

To summarize, the outlined methodology, although not optimal in a global sense, is capable of estimating the main mission parameters (minimum flight time, number of revolutions around the sun, and the minimum sun's distance) without the need of resorting to long numerical simulations. Its effectiveness is discussed in the next section.

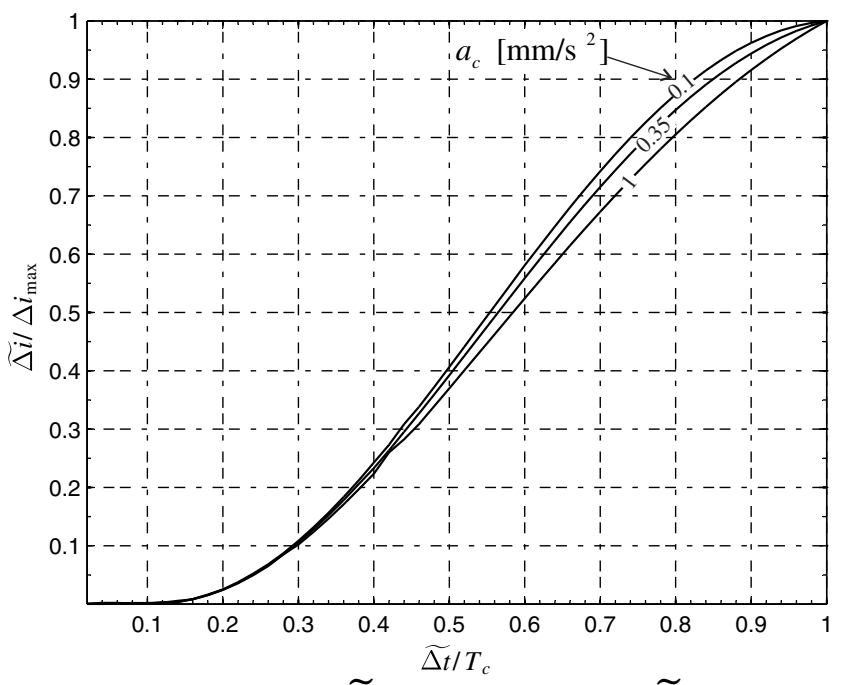

Fig. 6 Value of $\widetilde{\Delta i}$ as a function of $a_{c}$ and $\widetilde{\Delta t}$.

\section{Case Study}

The previous approximate method is now applied to the analysis of the SPI mission and the results are compared to the optimal solution found by Dachwald et al. in []] (note, however, that []] takes into account the actual eccentricity of the terrestrial heliocentric orbit).

The main mission parameters have been calculated for different values of the maximum admissible temperature in the range $\Theta_{\max } \in[460,580] \mathrm{K}$. The corresponding results are summarized in Fig. 7 along with the data taken from [6]. Figure 7 shows an excellent agreement between the results obtained with the approximate method and those of [6] as long as the trends of both $\Delta t$ and $r_{c}$ are concerned. In particular, the percentage difference in $\Delta t$ is less than $4 \%$, while in $r_{c}$ it is about $10 \%$.

Note that the time lengths of phases 1 and 2 are comparable for small values of $\Theta_{\max }$. On the other hand, as $\Theta_{\max }$ increases, the length of the cranking phase tends to reduce remarkably with respect to phase 1. In fact, an increase in the admissible value of $\Theta_{\max }$ guarantees the possibility of reducing the cranking radius. This implies, on one side, that the sailcraft can rotate the orbital plane more quickly (because the solar radiation pressure is greater) but, on the other side, that the transfer time necessary to reach a closer approach to the sun increases. Accordingly, the time $\Delta t_{3}$ required to reach the final orbit tends to increase as well. The latter remark has important consequences on the choice of dividing the whole transfer into three distinct phases. This matter is discussed in the next section.

\section{How Many Phases?}

So far the sailcraft trajectory has been studied under the assumption of three distinct phases. Although in a globally optimal solution the inclination change would happen with continuity along the whole mission [6, 11] , the proposed simplified strategy of phase division has been adopted here for the sake of reducing the number of parameters necessary to identify each phase and, therefore, to obtain a fully (albeit approximate) characterization of the mission performance. Having clarified that this approach is functional to obtain a suboptimal solution with a minimum computational effort, and that the phase division has a certain degree of arbitrariness, the question arises of whether other strategies may provide better solutions. We confine our study to the analysis of another strategy.
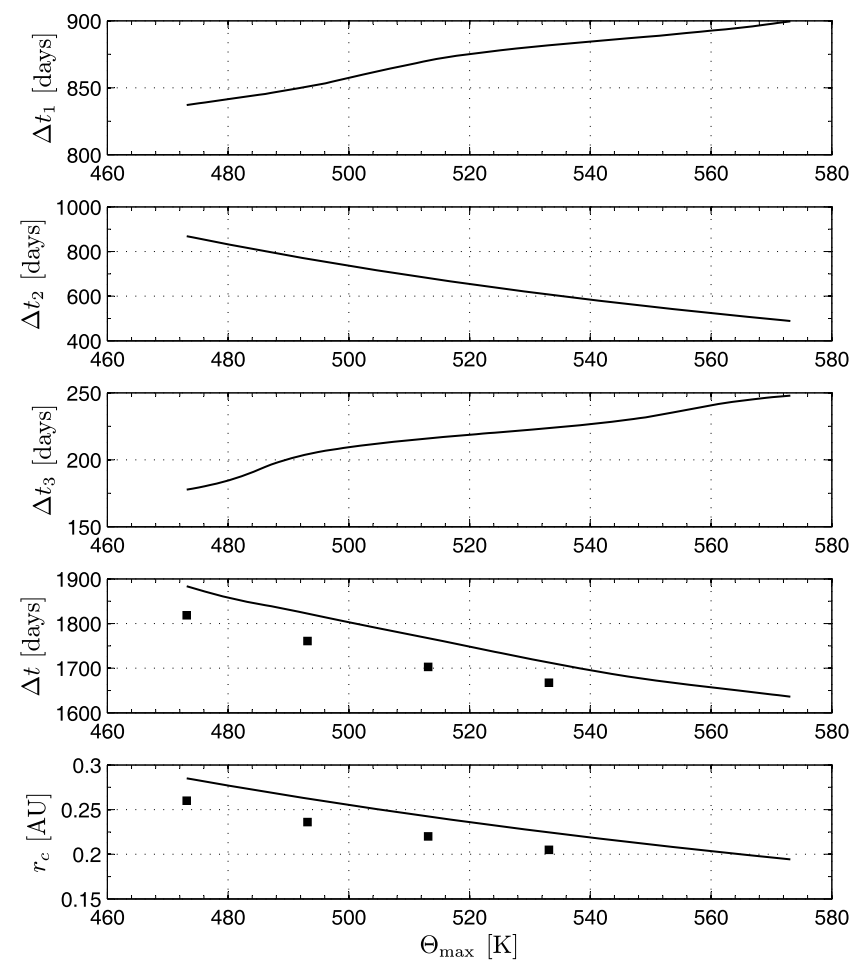

Fig. 7 Performance of SPI mission ( $\square$ data taken from [] $]$ ). 
The most simple alternative consists of achieving the transfer by rotating the orbital plane when the sun-sailcraft distance is exactly equal to $r_{f}$. In this case the trajectory would be constituted by two phases only, the first one being a two-dimensional, circle-to-circle transfer between $r_{0}$ and $r_{f}$. The performance corresponding to this strategy that, for the sake of clarity, will be referred to as a doublephase transfer (in contrast to the previous triple-phase transfer), can be easily calculated with the aid of the preceding results discussed for phases 1 and 2. Clearly, the use of a triple-phase transfer with a cranking orbit $r_{c}<r_{f}$ implies, when compared to the double-phase strategy, a decrease in the cranking time and, at the same time, both an increase in the length of phase 1 and the appearance of an additional third phase. Therefore, it is reasonable to investigate which strategy is better (that is, closer to an optimal result) as a function of the desired value of $i_{f}$.

It can be shown that there exists a critical value of the plane change, referred to as $\Delta i^{\star}$, such that if $i_{f}-i_{0}>\Delta i^{\star}$, a triple-phase mission is advisable as it requires a total mission time shorter than that for a double-phase strategy. This critical value, that can be calculated by simulation, is a function of $\Theta_{\max }, r_{f} / r_{0}$, and $a_{c}$. As an example, consider the main SPI mission parameters, that is, $a_{c}=0.35 \mathrm{~mm} / \mathrm{s}^{2}, \quad r_{0}=1 \mathrm{AU}, \quad r_{f}=0.48 \mathrm{AU}, \quad$ and $\Theta_{\max }=$ $513.15 \mathrm{~K}$. Figure 8 compares the two strategies for different values of final inclination in the range $i_{f} \in(0,90] \mathrm{deg}$. The ripples in the figure, in accordance with the same behavior shown in $[6,11]$, are due to the fact that the inclination rate of change is variable during the cranking trajectory, because the propelling acceleration is more effective near the orbital nodes.

Figure 8 confirms the existence of a critical value of inclination $i_{f} \cong 24.5^{-}$deg beyond which a triple-phase strategy has better performance. In particular, assuming an inclination of $i_{f}=75 \mathrm{deg}$ (equal to that of the SPI mission), there is a remarkable difference in flight time (794 days) between the values estimated with a triplephase and a double-phase strategy. This difference reduces with the decrease of the maximum admissible temperature. For example, assuming a value $\Theta_{\max }=373.15 \mathrm{~K}$ (corresponding to the "cold mission scenario" of [6]), the critical value of inclination is $\Delta i^{\star} \simeq$ $43 \mathrm{deg}$ (see Fig. 8), while the flight times for the two mission strategies are very close, equal to 2564 days and 2511 days. The physical interpretation for this behavior is that the cranking distance $r_{c}$ is connected to the maximum admissible sail temperature through Eq. (28). In fact, when $\Theta_{\max }=373.15 \mathrm{~K}$, Eq. (28) gives $r_{c} \simeq 0.455 \mathrm{AU}$, a value corresponding to about $95 \%$ of the final orbit radius $\left(r_{f}=0.48 \mathrm{AU}\right)$. A high value of $r_{c} / r_{f}$ implies that the sail is not able to exploit the solar pressure radiation increase (when

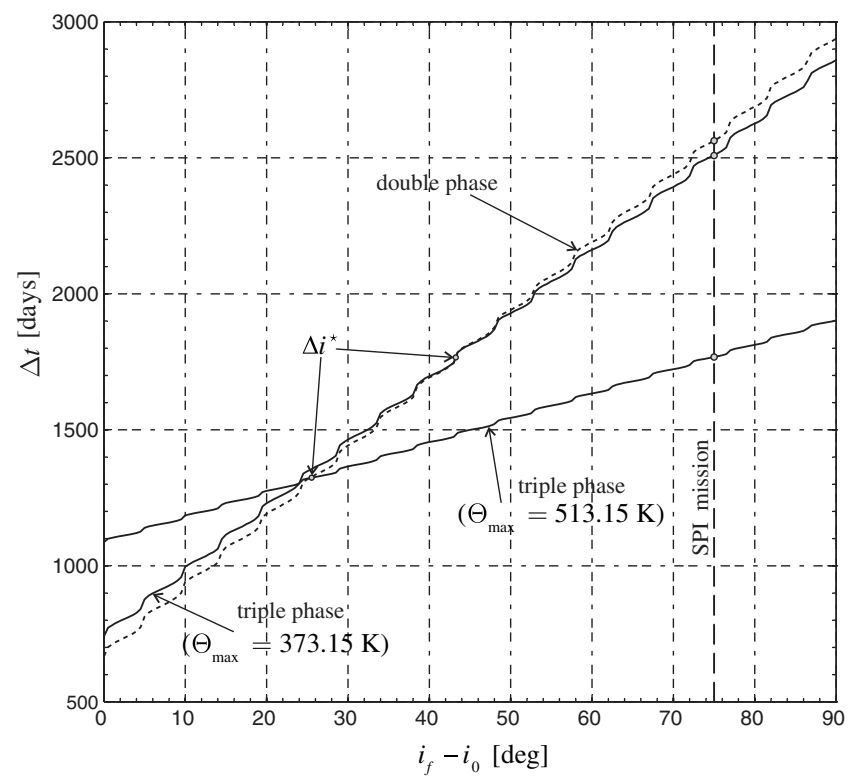

Fig. 8 Performance comparison of double-phase vs triple-phase mission strategies $\left(a_{c}=0.35 \mathrm{~mm} / \mathrm{s}^{2}, r_{0}=1 \mathrm{AU}\right.$, and $\left.r_{f}=0.48 \mathrm{AU}\right)$.

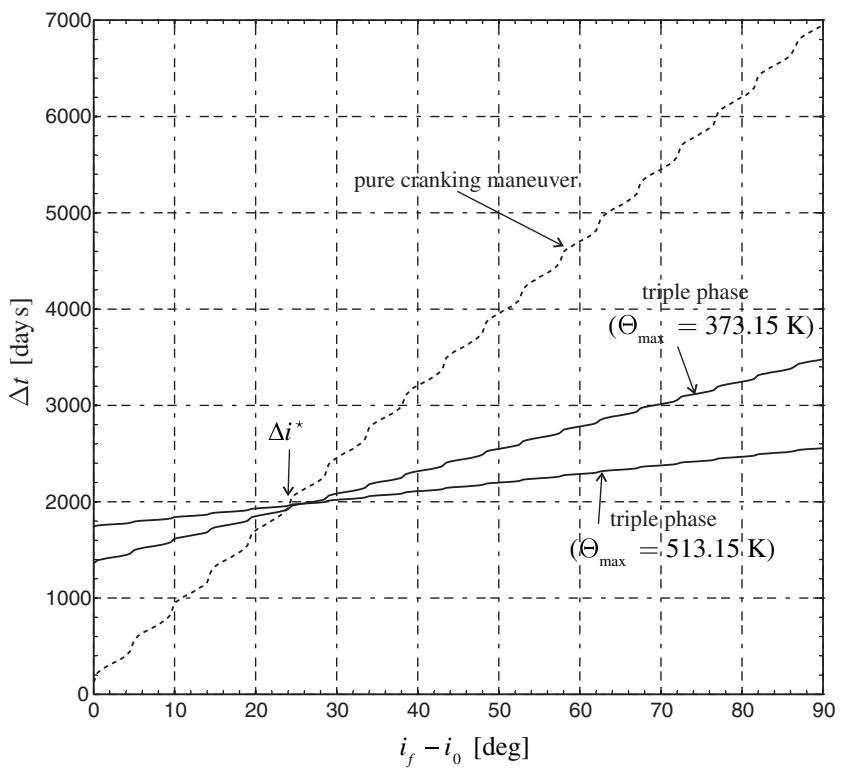

Fig. 9 Parking orbit cranking: single-phase vs triple-phase mission strategies $\left(a_{c}=0.35 \mathrm{~mm} / \mathrm{s}^{2}\right.$, and $\left.r_{f} \equiv r_{0}=1 \mathrm{AU}\right)$.

compared to that measured at a distance $r_{f}$ from the sun) to speed up the cranking maneuver. This, in turn, implies a substantial coincidence between the two mission strategies as long as the mission time is concerned.

Another possible application of the proposed methodology involves the estimate of the transfer times to highly inclined nearEarth asteroids. In particular, Fig. 9 shows the performance of a solar sail (with $a_{c}=0.35 \mathrm{~mm} / \mathrm{s}^{2}$ ) whose aim is to rotate the parking orbit plane of a given angle $i_{f}$. In this special case, because $r_{f} \equiv r_{0}=1 \mathrm{AU}$, the double-phase strategy coincides with a pure cranking maneuver.

Figure 9 shows that a pure cranking maneuver is better than a triple-phase strategy for small values of the inclination angle $\left(i_{f}<15 \mathrm{deg}\right)$, while the reverse holds for high orbit inclinations $\left(i_{f}>30 \mathrm{deg}\right)$. In this latter case $\Delta i^{\star} \cong 25 \mathrm{deg}$, a value nearly independent of $\Theta_{\max }$.

\section{Conclusions}

The problem of solar sail orbital transfer with plane change has been analyzed with a systematic approach. Assuming that the starting and arrival orbits are both circular, the minimum time transfer can be approximated with a near-optimal solution capable of estimating the globally optimal results with small percentage errors. To this end, the whole trajectory is first divided into two or three phases, depending on the value of the inclination change between the initial and final orbit. Each phase can be fully characterized by solving a minimum time problem with an indirect approach and the results have been collected in graphs and fitting functions. The final trajectory is then approximated by simply joining the different phases. As a result, the whole calculation is immediate and requires no simulation effort. The solutions found are useful for a preliminary mission analysis as well as to conduct trade-off studies. The obtained results can also been used as a guide for finding true globally optimal trajectories and as a first guess for more refined optimization algorithms. Important applications of this method are related to the performance analysis of missions to and between near-Earth objects with highly inclined orbits, or for the observation of sun's polar regions. A comparison with globally optimal results found in the literature for the Solar Polar Imager mission confirms the effectiveness of the proposed method.

\section{References}

[1] McInnes, C. R., Solar Sailing: Technology, Dynamics and Mission Applications, Springer-Praxis Series in Space Science and Technology, Springer-Verlag, Berlin, 1999, pp. 46-54, 136-151. 
[2] Macdonald, M., Hughes, G. W., McInnes, C. R., Lyngvi, A., Falkner, P., and Atzei, A., "Solar Polar Orbiter: A Solar Sail Technology Reference Study," Journal of Spacecraft and Rockets, Vol. 43, No. 5, Sept.-Oct. 2006, pp. 960-972. doi: $10.2514 / 1.16408$

[3] Dachwald, B., Seboldt, W., and Richter, L., "Multiple Rendezvous and Sample Return Missions to Near-Earth Objects Using Solar Sailcraft," Acta Astronautica, Vol. 59, No. 8-11, 2006, pp. 768-776. doi:10.1016/j.actaastro.2005.07.061

[4] Burch, J. L., "Sun-Earth Connection Roadmap: Strategic Planning for the Years 2000-2020," TR, National Aeronautics and Space Administration, April 1997.

[5] Fisher, R. R., "Heliophysics: The New Science of the Sun-Solar System Connection," TR, National Aeronautics and Space Administration, Feb. 2006, http://sec.gsfc.nasa.gov/Roadmap_FINALpri.pdf [cited 11 Dec. 2008].

[6] Dachwald, B., Ohndorf, A., and Wie, B., "Solar Sail Trajectory Optimization for the Solar Polar Imager (SPI) Mission," AIAA Paper 2006-6177, 2006.

[7] Hughes, G. W., and McInnes, C. R., "Solar Sail Hybrid Trajectory Optimization for Non-Keplerian Orbit Transfers," Journal of Guidance, Control, and Dynamics, Vol. 25, No. 3, May-June 2002, pp. 602-604. doi:10.2514/2.4924

[8] Cichan, T., and Melton, R. G., "Optimal Trajectories for Non-Ideal Solar Sails," AAS Paper 01-471, 2001.

[9] Melton, R. G., "Comparison of Direct Optimization Methods Applied to Solar Sail Problems," AIAA Paper 2002-4728, 2002.

[10] Sauer, C. G., Jr., "Optimum Solar-Sail Interplanetary Trajectories," AIAA Paper 76-792, 1976.

[11] Sauer, C. G., Jr., "Solar Sail Trajectories for Solar Polar and Interstellar Probe Missions," AAS Paper 99-336, 1999.

[12] Mengali, G., and Quarta, A. A., "Optimal Three-Dimensional Interplanetary Rendezvous Using Nonideal Solar Sail," Journal of Guidance, Control, and Dynamics, Vol. 28, No. 1, Jan.-Feb. 2005, pp. 173-177. doi: $10.2514 / 1.8325$

[13] Sauer, C. G., Jr., "A Comparison of Solar Sail and Ion Drive Trajectories for a Halley's Comet Rendezvous Mission," AAS Paper 77-104, 1977.

[14] Dachwald, B., and Seboldt, W., "Solar Sailcraft of the First Generation Mission Applications to Near-Earth-Asteroids," 54th International Astronautical Congress, IAC-03-Q.5.06, Bremen, Germany, Sept.Oct. 2003

[15] Betts, J. T., "Survey of Numerical Methods for Trajectory Optimization," Journal of Guidance, Control, and Dynamics, Vol. 21, No. 2, March-April 1998, pp. 193-207. doi: $10.2514 / 2.4231$

[16] Macdonald, M., and McInnes, C. R., "Analytical Control Laws for Planet-Centered Solar Sailing," Journal of Guidance, Control, and Dynamics, Vol. 28, No. 5, Sept.-Oct. 2005, pp. 1038-1048. doi: $10.2514 / 1.11400$

[17] Mengali, G., and Quarta, A. A., "Near-Optimal Solar-Sail OrbitRaising from Low Earth Orbit," Journal of Spacecraft and Rockets,
Vol. 42, No. 5, Sept.-Oct. 2005, pp. 954-958. doi: $10.2514 / 1.14184$

[18] Mengali, G., and Quarta, A. A., "Earth Escape by Ideal Sail and SolarPhoton Thrustor Spacecraft," Journal of Guidance, Control, and Dynamics, Vol. 27, No. 6, Nov.-Dec. 2004, pp. 1105-1108. doi:10.2514/1.10637

[19] Kluever, C. A., "Simple Guidance Scheme for Low-Thrust Orbit Transfers," Journal of Guidance, Control, and Dynamics, Vol. 21, No. 6, Nov.-Dec. 1998, pp. 1015-1017. doi: $10.2514 / 2.4344$

[20] Rowe, W. M., Luedke, E. E., and Edwards, D. K., "Thermal Radiative Properties of Solar Sail Film Materials," AIAA Paper 78-852, 1978.

[21] Stimpson, L. D., Greenfield, M. L., Jaworski, W., and Wolf, F., "Thermal Control of a Solar Sail," AIAA Paper 78-885, 1978.

[22] Wright, J. L., Space Sailing, Gordon and Breach Science Publisher, Berlin, 1992, pp. 223-226.

[23] Walker, M. J. H., Owens, J., and Ireland, B., "A Set of Modified Equinoctial Orbit Elements," Celestial Mechanics, Vol. 36, Aug. 1985, pp. 409-419. doi:10.1007/BF01227493

[24] Walker, M. J., "Erratum-A Set of Modified Equinoctial Orbit Elements," Celestial Mechanics, Vol. 38, April 1986, pp. 391-392. doi:10.1007/BF01238929

[25] Betts, J. T., "Very Low-Thrust Trajectory Optimization Using a Direct SQP Method," Journal of Computational and Applied Mathematics, Vol. 120, No. 1, Aug. 2000, pp. 27-40. doi:10.1016/S0377-0427(00)00301-0

[26] Coverstone, V. L., and Prussing, J. E., "Technique for Escape from Geosynchronous Transfer Orbit Using a Solar Sail," Journal of Guidance, Control, and Dynamics, Vol. 26, No. 4, July-Aug. 2003, pp. 628-634. doi: $10.2514 / 2.5091$

[27] Dachwald, B., Mengali, G., Quarta, A. A., and Macdonald, M., "Parametric Model and Optimal Control of Solar Sails with Optical Degradation," Journal of Guidance, Control, and Dynamics, Vol. 29, No. 5, Sept.-Oct. 2006, pp. 1170-1178. doi: $10.2514 / 1.20313$

[28] Bryson, A. E., and Ho, Y. C., Applied Optimal Control, Chap. 2, Hemisphere Publishing Corporation, New York, 1975, pp. 71-89.

[29] Forsythe, G. E., Michael, A. M., and Moler, C. B., Computer Methods for Mathematical Computations, Prentice-Hall, Englewood Cliffs, NJ, 1977, pp. 192-235.

[30] Alfano, S., and Thorne, J. D., "Circle-to-Circle Constant-Thrust Orbit Raising," Journal of the Astronautical Sciences, Vol. 42, No. 1, Jan.March 1994, pp. 35-45.

[31] Mengali, G., and Quarta, A. A., "Solar-Sail-Based Stopover Cyclers for Cargo Transportation Missions," Journal of Spacecraft and Rockets, Vol. 44, No. 4, July-Aug. 2007, pp. 822-830. doi: $10.2514 / 1.24423$

[32] McInnes, C. R., Hughes, G., and Macdonald, M., "Payload Mass Fraction Optimization for Solar Sail Cargo Missions," Journal of Spacecraft and Rockets, Vol. 39, No. 6, Nov.-Dec. 2002, pp. 933-935. doi: $10.2514 / 2.3901$ 